\title{
A phantom study on bladder and rectum dose measurements in brachytherapy of cervix cancer using FBX aqueous chemical dosimeter
}

\author{
Anil K. Bansal ${ }^{a}$, Manoj K. Semwal ${ }^{b, *}$, Deepak Arora ${ }^{a}$, \\ D.N. Sharma ${ }^{\text {c }}$, P.K. Julka ${ }^{\text {C }, ~ G . K . ~ R a t h ~}{ }^{\text {c }}$
}

a Department of Radiation Oncology, Max Hospital, Saket, New Delhi 110017, India

b Department of Radiotherapy, Army Hospital (Research \& Referral), Delhi Cantonment, New Delhi 110010, India

' Department of Radiotherapy, Institute Rotary Cancer Hospital, All India Institute of Medical Sciences, New Delhi 110029, India

Received 6 February 2012; received in revised form 17 April 2012; accepted 17 May 2012 Available online 9 June 2012

\section{KEYWORDS \\ FBX dosimeter; \\ Brachytherapy; \\ Phantom \\ measurements}

\begin{abstract}
The ferrous sulphate-benzoic acid-xylenol orange (FBX) chemical dosimeter, due to its aqueous form can measure average volume doses and hence may overcome the limitations of point dosimetry. The present study was undertaken to validate the use of FBX dosimeter for rectum and bladder dose measurement during intracavitary brachytherapy (ICBT) and transperineal interstitial brachytherapy (TIB). We filled cylindrical polypropylene tubes (PT) and Foley balloons (FB) with FBX solution and used them as substitutes for rectum and bladder dose measurements respectively. A water phantom was fabricated with provision to place the Fletcher-type ICBT and MUPIT template applicators, and FBX filled PT and FB within the phantom. The phantom was then CT scanned for treatment planning and subsequent irradiation. Our results show that the average difference between DVH derived dose value and FBX measured dose is $3.5 \%$ (PT) and $13.7 \%$ (FB) for ICBT, and $9 \%(\mathrm{PT})$ and $9.9 \%(\mathrm{FB})$ for TIB. We believe that the FBX system should be able to provide accuracy and precision sufficient for routine quality assurance purposes. The advantage of the FBX system is its water equivalent composition, average volume dose measuring capability, and energy and temperature independent response as compared to TLD or semiconductor dosimeters. However, detailed studies
\end{abstract}

\footnotetext{
* Corresponding author. Tel.: +911125691181.

E-mail address: manojsemwal@yahoo.co.in (M.K. Semwal).
}

1120-1797/\$ - see front matter (c) 2012 Associazione Italiana di Fisica Medica. Published by Elsevier Ltd. All rights reserved. doi:10.1016/j.ejmp.2012.05.005 
will be needed with regards to its safety before actual in-vivo dose measurements are possible with the FBX dosimeter.

(c) 2012 Associazione Italiana di Fisica Medica. Published by Elsevier Ltd. All rights reserved.

\section{Introduction}

Intracavitary brachytherapy (ICBT)/transperineal interstitial brachytherapy (TIB) in combination with external beam radiotherapy (EBRT) is generally the standard of care for cervical cancer especially in advanced stages. With the increasing use of high-dose-rate (HDR) brachytherapy there is a concern for increased potential of late-effect toxicities to organs at risk (OAR) mainly the bladder and rectum [1]. Estimation and reporting of dose received by OAR in HDR brachytherapy is therefore crucial for taking corrective actions during treatment and also for assessment of late toxicities. The dose prescription and reporting protocols have changed over the years with changing imaging technology and planning tools for better correlation with the clinical outcome. An important change in this direction is the increasing use of 3-D volume dose estimation and reporting [2-4] as compared to the earlier point-based dose estimation recommended by the International Commission on Radiation Units and Measurements [5,6]. Even with imagebased 3D planning, a number of factors could still lead to a difference in the predicted dose and the dose actually delivered to the patient. Some of these factors are CT image quality affecting the accuracy of the reconstruction of the patient clinical structures and implant catheters, the accuracy of the treatment planning system algorithm in dose calculations, the source calibration uncertainty, the accuracy in the realization of the planned source dwell position and time during delivery, source transit time, change in organ position and shape that occurs in between imaging used for planning and actual treatment due to shift of patient and/or difference in bladder and rectum filling. The possibility of human error, especially with regard to correct connection of catheters to the afterloader is another crucial factor that must also be taken into account [7].

In-vivo dosimetry is a useful tool both in evaluating the actual doses to rectum and bladder, and in avoiding treatment misadministrations. It should be performed in addition to-and not instead of 3D dose computations at the reference points and other points of interest. Thermoluminescence dosimeters (TLDs) and semiconductor diodes have been commonly used by many groups for this purpose [8-12]. These dosimeters are used for point dose measurements and hence suffer from the usual drawback namely that the measured dose may not always represent the clinically significant dose to the OAR. Hence repetition and measurements at several points may be needed.

The ferrous sulphate-benzoic acid-xylenol orange (FBX) aqueous chemical dosimeter is a modification of the wellknown Fricke chemical dosimeter [13]. It has some favorable properties as compared to other dosimeters such as its energy (for $\mathrm{x}$-rays and gamma-rays from 33 to $1250 \mathrm{keV}$ ), and temperature (from 15 to $45^{\circ} \mathrm{C}$ ) independent response. Some of the limitations of the FBX system are the seemingly cumbersome and labour intensive procedure of handling the chemicals, and large size of the dosimeter. Also, if FBX is considered for in-vivo dosimetry, its toxicity profile will need to be addressed in detail. Due to its lower dose detection threshold compared to Fricke system it has been studied for many applications in radiotherapy. For example, FBX system has been used for percentage depth dose measurements, machine output calibrations, virtual wedge profile measurements, in-vivo dosimetry in external beam radiotherapy [14-18] and brachytherapy source calibration [19]. The use of FBX dosimeter has so far not been reported for bladder and rectal dosimetry. We believe that because of its aqueous form, it may be useful in measuring average volume dose especially in irregularly-shaped structures. The present phantom study is aimed at exploring the potential of the FBX dosimeter for rectum and bladder dose measurement during ICBT and TIB.

\section{Material and methods}

\section{FBX dosimeter}

The FBX dosimetry comprises of three main steps namely preparation of the FBX solution, irradiation of FBX filled vials/ cells, and optical densitometry. The FBX solution was prepared following the method described by Gupta et al. [14] to achieve the following composition: $0.2 \mathrm{~mol} \mathrm{~m}^{-3}$ ferrous ammonium sulphate, $5.0 \mathrm{~mol} \mathrm{~m}^{-3}$ benzoic acid, and $0.2 \mathrm{~mol} \mathrm{~m}^{-3}$ xylenol orange in $40.0 \mathrm{~mol} \mathrm{~m}^{-3}$ sulfuric acid. All the chemicals were analytical grade and came from E. Merck (Germany) except for xylenol orange which came from Loba Chemie (Austria). Readily available cylindrical polypropylene tubes (PT) of length approximately $5.5 \mathrm{~cm}$ and diameter $1.1 \mathrm{~cm}$ filled with FBX solution were used for rectal dose measurement.

\section{Calibration}

The irradiation for FBX calibration was carried out on a Theratron 780C telecobalt machine (Theratronics, Canada). The calibration for PT was in the dose range 2-10 Gy, in steps of $2 \mathrm{~Gy}$, and that for Si Balloon was in the range $2-8 \mathrm{~Gy}$, in steps of $2 \mathrm{~Gy}$, with one additional irradiation at $3 \mathrm{~Gy}$. The telecobalt output was measured using a Farmer-type ion-chamber (PTW, Germany) whose calibration, in turn, was traceable to the national secondary standards dosimetry laboratory. An in-house fabricated water phantom having dimensions $30 \mathrm{~cm} \times 30 \mathrm{~cm} \times 15 \mathrm{~cm}$ was used for the irradiation of FBX filled PTs and Foley balloons. The optical density (OD) of the irradiated samples was measured with a five filter digital colorimeter (Aimil, India) at $540 \mathrm{~nm}$ at least $30 \mathrm{~min}$ after irradiation against unirradiated blank solution following the standard procedure [14]. The calibration process was repeated three times with new FBX solution each time for better assessment of reproducibility. For bladder dose measurements, Foley balloons (FBs) of different make, as available in the market, were initially investigated for suitability in terms 
of reproducibility and stability. We found out that FBs of different material composition and makes varied widely in terms of reproducibility and stability. Our initial trials indicated that Rusch (Malaysia) make FBs performed better as compared to the FBs of other makes. Further investigations were therefore confined to Rusch-make FBs composed of either silicone rubber or pure silicone. The procedure for filling-up and extraction of FBX solution in the FBs was standardized to improve the reproducibility. A glass syringe (10 cc volume) with glass piston instead of plastic syringes with rubber piston-head was used for filling-up and extraction of FBX solution to reduce contamination/reaction related uncertainties. Post irradiation, the extraction of solution was carried out in two steps; first about $3 \mathrm{ml}$ solution was extracted and discarded and then the remaining solution was extracted and transferred directly into the measurement tube (cell) of the colorimeter for OD measurement. FBs from three lots were used for investigating the lot to lot variations.

\section{ICBT and TIB irradiation}

For ICBT and TIB, CT compatible Fletcher-type applicator and MUPIT template applicator (Nucletron, The Netherlands) respectively were securely fixed in the phantom along with the FBX filled PT and Foley balloon (Figs 1 and 2). The phantom set-up was scanned on a CT scanner model Volume Zoom (Siemens, Germany) with $2.5 \mathrm{~mm}$ slice thickness, before filling with water for irradiation, for better delineation of PT and FB as part of the treatment planning process. The scans were pushed to Plato brachytherapy treatment planning system Vers 14.3.7 (Nucletron, The Netherlands) for contouring and treatment planning. Standard treatment plans were created to deliver $7 \mathrm{~Gy}$ dose at point ' $A$ ' for ICBT and at $5 \mathrm{~mm}$ box surface around the implant for TIB and pushed to the microSelectron high dose rate brachytherapy machine (Nucletron, The Netherlands) for irradiation. For ICBT, the position of the os on the CT image was identified by the cervical marker ring placed on the tandem. Point ' $A$ ' was

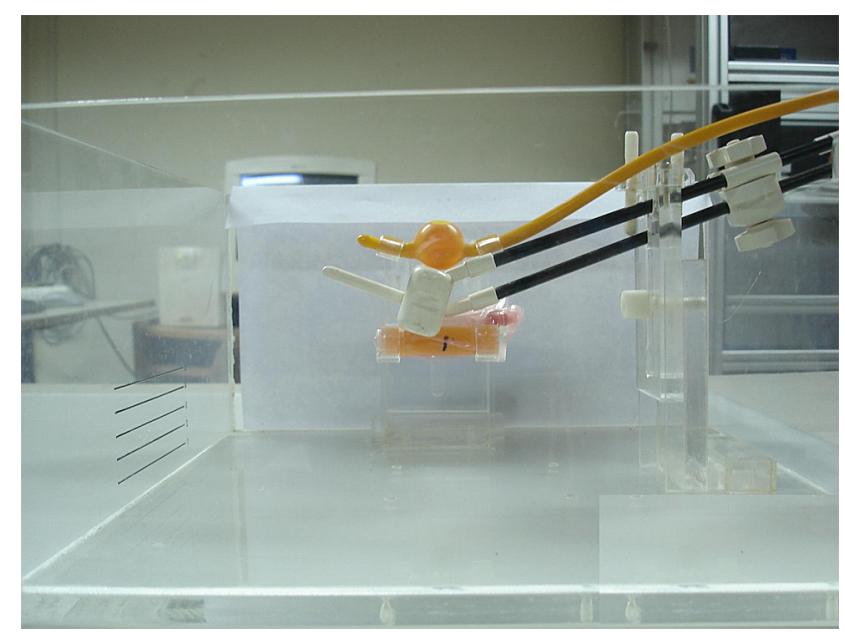

Figure 1 Phantom set-up side-on photograph. Foley balloon and polypropylene tube filled with FBX solution placed inside the water phantom with the Fletcher-type brachytherapy applicator for planning and irradiation.

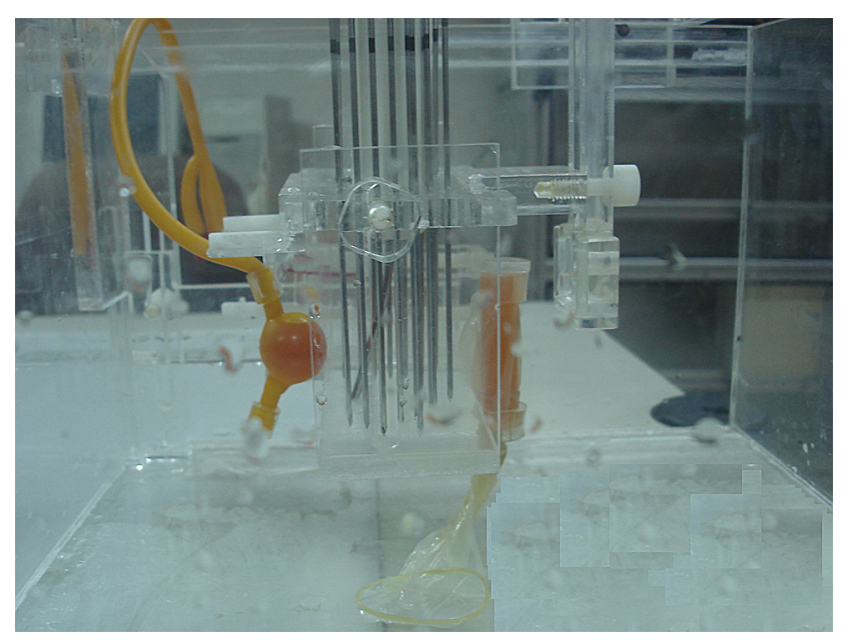

Figure 2 Phantom set-up side-on photograph. Foley balloon and polypropylene tube filled with FBX solution placed inside the water phantom with the MUPIT brachytherapy applicator system for planning and irradiation.

found relative to the os marker, first by measuring $2 \mathrm{~cm}$ superiorly along the tandem, and from that point moving $2 \mathrm{~cm}$ perpendicular to the tandem in the lateral direction. The active dwell positions were kept at 1, 3, 5, 7, 10, 13, 16 from the tip for uterine tandem and at 3-6 (continuous four dwell positions) for the colpostats with $2.5 \mathrm{~mm}$ step in such a way that these positions were bisected by the uterine tandem. For TIB, from the os upto $4 \mathrm{~cm}$ length of the needles were loaded towards the tip and $2 \mathrm{~cm}$ towards the open end (total $6 \mathrm{~cm}$ length). First and last dwell positions were at $2.5 \mathrm{~mm}$ step size with in between dwell positions at $5 \mathrm{~mm}$ step size for all needles in TIB. The relative positions of the FB and PT with respect to the sources were kept as commonly encountered in clinical practice (about $2 \mathrm{~cm}$ from the sources). Figure 3 showing CT cross-section has been added for better appreciation of the geometry. The OD measurement of the irradiated FBX samples was carried out on the colorimeter at $540 \mathrm{~nm}$. The entire process of dose measurement was repeated five times for both ICBT and TIB. Care was taken to ensure that FBs from the same batch were used for calibration and dose estimation. Subsequently, the dose measured was compared with the dose computed from the TPS using dose volume histogram (DVH) data.

\section{Results and discussion}

Figure 4 shows calibration graphs for PT and silicone FB dosimeters. As can be seen, the dose versus optical density response is linear for both the dosimeters with almost identical slopes indicating that the sensitivity is similar for both the PT and the silicone FB dosimeter. The reproducibility along with minimum measurable dose for PT, silicone FB and silicone-rubber FB is shown in Table 1 . The reproducibility values quoted in the table are at $4 \mathrm{~Gy}$ level. It is observed that PT has minimum uncertainty (standard error) and silicone-rubber FB has this value as maximum. In terms of sensitivity, PT and silicone-FB have similar sensitivity (OD per unit dose) as compared to silicone-rubber FB which has lesser value of OD per unit dose. The value of minimum 


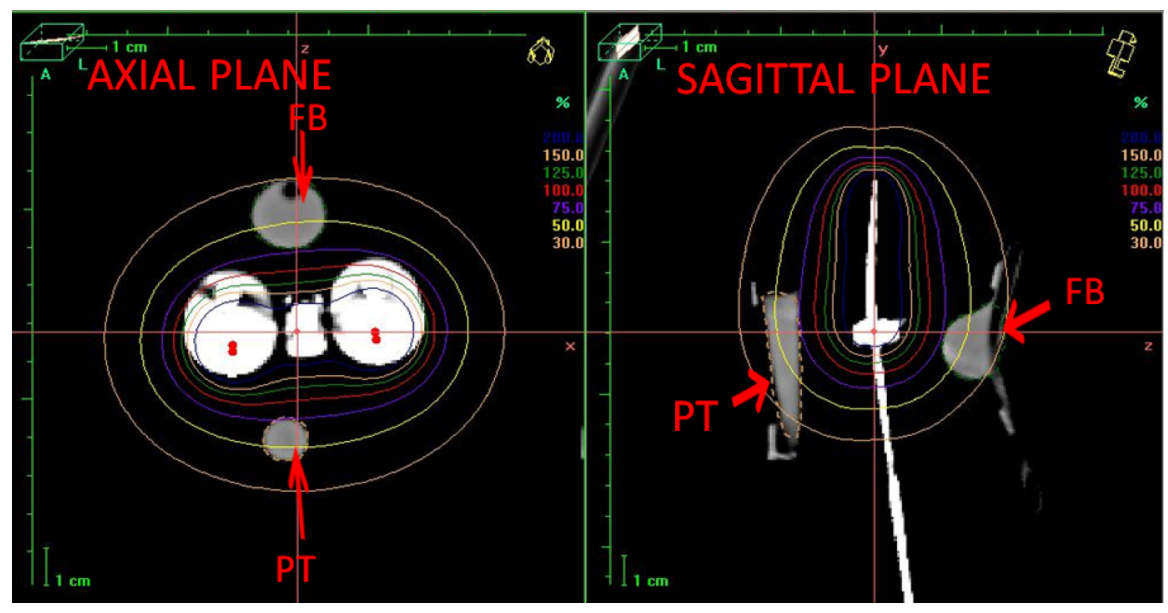

Figure 3 "Snapshot" showing axial and sagittal CT sections of a typical irradiation plan on the treatment planning system. The Fletcher-type applicator, Foley Balloon (FB), and polypropylene tube (PT) filled with FBX solution are clearly visible.

measurable dose (MMD) is comparable for PT and silicone$\mathrm{FB}$ but is high for silicone-rubber FB. The MMD was estimated using the equation $M M D=2 \times \sigma_{\mathrm{B}} / \phi$, where $\sigma_{\mathrm{B}}$ is standard deviation for blank FBX solution OD values, and $\phi$ is sensitivity (OD per unit dose) [20]. Further, the maximum variation in response between two lots of silicone-FB was observed to be $12.8 \%$. It is evident from the results that silicone-FB is better suited for dosimetry as compared to silicone-rubber FB.

Table 1 also shows the difference between calculated and measured doses as percentage of calculated dose with PT and silicone-FB for both ICBT and TIB. The calculated doses shown are DVH based volume average doses for both PT and FB. In ICBT, the average difference between calculated and measured dose is $3.5 \%$ (range $1.1-5.8 \%$ ) for PT and $13.7 \%$ (range 10.4-15.1\%) for silicone-FB. In TIB, the average difference is $9 \%$ (range $2.1-13.1 \%$ ) and 9.9\% (range 5.8-14.6\%) for PT and silicon-FB respectively. The measured dose ranged from 2.3 to $3.7 \mathrm{~Gy}$ for all categories of measurements. The differences observed between the measured and calculated

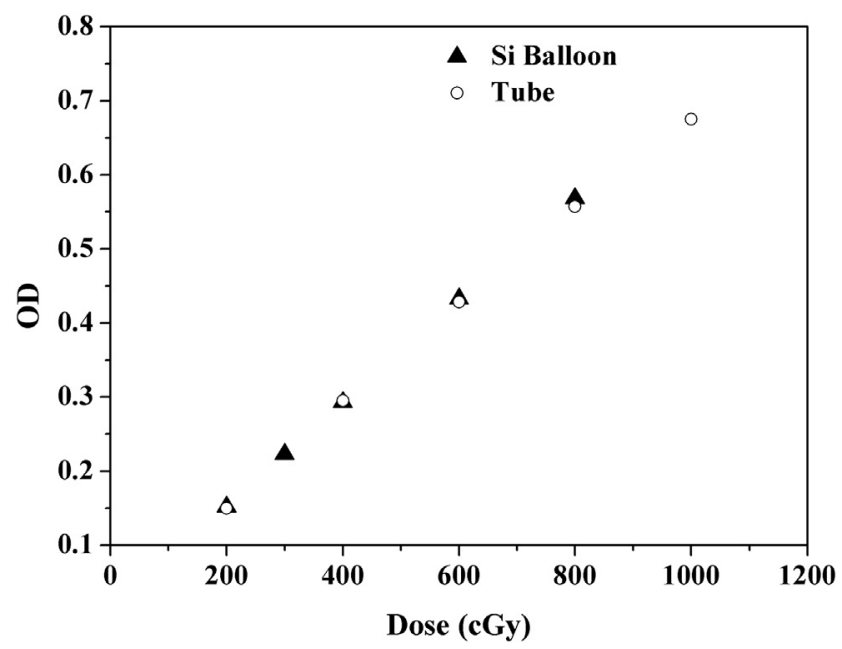

Figure 4 Calibration graph showing dose (cGy) versus optical density (OD) for polypropylene tube (Tube) and Silicone Foley balloon (Si Balloon) FBX dosimeters. doses could be due to the fact that i) the term "average" used in the context of calculated dose from the DVH values does not exactly correspond to the "average" dose as measured in an aqueous medium. In the former case the single value (average calculated dose) is estimated by averaging the calculated doses for a large number of points within a contoured volume whereas in the latter the measurement itself produces a single value. It is then assumed that the measuring medium being aqueous, the measured single value represents the "average measured dose" for the volume of the dosimeter ii) the reproducibility or uncertainty error (larger in the case of FBX), and the other errors associated with FBX measurements are different as compared to those associated with calculation based methods". The smaller difference between calculated and measured doses for PT as compared to FB could be due to the fact that i) The PT, as shown Figs. 1 and 2, is placed in the phantom in such a way that its smaller dimension (diameter $1.1 \mathrm{~cm}$ ) is along the direction of maximum dose fall-off which is non-linear. Therefore, we believe that the dose measured with it should be closer to the average calculated dose as compared to FB (2 cm diameter) ii) reproducibility of PT (2\%) is better than that of FB (3.9\%) as was shown earlier, and iii) the CT scans were performed before filling the phantom with water. Subsequent filling of the phantom with water for irradiation could result in small deformation in the shape of the submerged $\mathrm{FB}$, unlike the rigid $\mathrm{PT}$, resulting in some difference in the volume used for average dose calculation and the one that was irradiated.

Stuecklschweiger et al. [8] in their patient study observed a maximum difference of $\pm 20 \%$ in the TLD measured and radiographically calculated dose for rectum. They attributed the deviations to both location and calibration errors. Alecu et al. [11] carried out in-phantom and in-vivo dosimetry with diodes in patients of carcinoma cervix treated by HDR brachytherapy. For the phantom study, by simulating the real clinical situation, they reported that the calculated values were within $5 \%$ of the measured values for the rectum. For patients, the average discrepancy between the measured and calculated doses was $11 \%$ with maximum being $15 \%$. Clark et al. [10] compared ICRU 38 reference points doses to bladder and rectum. For the rectum reference point the calculated dose 
Table 1 Comparison of FBX-measured and calculated doses.

\begin{tabular}{lllllc}
\hline FBX cell type & MMD (cGy) & SE (\%) & OD/Gy & $\begin{array}{l}\text { Difference for ICBT } \\
\text { (Mean } \pm \text { std dev) }\end{array}$ & Difference for TIB (Mean \pm std dev) \\
\hline Si-Rubber FB & 6.7 & 8.8 & 0.06 & $25.5 \pm 4.3$ & $30.7 \pm 3.3$ \\
Si-FB & 4.9 & 3.9 & 0.073 & $13.7 \pm 1.9$ & $9.9 \pm 4.4$ \\
PT & 5.6 & 2.0 & 0.072 & $3.5 \pm 1.6$ & $9 \pm 3.9$ \\
\hline
\end{tabular}

$M M D=$ Minimum measurable dose; SE = Standard error; OD = Optical density; Difference = Difference between measured and calculated dose values shown as percentage of calculated value; ICBT and TIB = Intracavitary and Transperineal brachytherapy; Si-Rubber FB = Silicone rubber Foley balloon; Si-FB = Silicone Foley balloon; PT = Polypropylene tube.

was $81 \%$ of the point ' $A$ ' dose and the measured dose was $28 \%$. For the bladder reference point the dose was $80 \%$ of the point ' $A$ ' dose and the measured dose was $37 \%$. In their phantom study using diodes, Waldhausl et al. [12] reported that difference between calculations and measurements was close to $5 \%$ (range -8 to $0 \%$ ). In patients, the difference between calculated and measured doses for rectum was $11 \%$ (range $-31-90 \%$ ) and for bladder 4\%(range -27 to $26 \%$ ). Using TLDs for in-vivo dose measurements in ICRT, Lahtinen et al. [9] reported a maximum deviation of $40 \%$ between point doses measured along the anterior rectal wall and calculated doses. For ICRU reference points the maximum deviation of $30 \%$ was observed by them. Toye et al. [21] also used TLDs for in-vivo dosimetry of the urethra and rectum in HDR brachytherapy of prostate. They found out that for urethra the percent difference in peak dose between the measured and the calculated dose for 53 patients ranged from $-34 \%$ to $66 \%$ with average difference of $6 \%$ (standard deviation $18 \%$ ). For the rectum, the difference ranged from $-36 \%$ to $101 \%$ with average difference of $20 \%$ (standard deviation 31\%).

The above quoted studies show that the variations between measured and calculated dose in in-vivo point dosimetry by diodes and TLDs, is $15 \%$ or more. A large part of these variations could be due to variation in location and calibration errors. A variation of even $1 \mathrm{~mm}$ in position of a point could result in deviation in dose of $\pm 10 \%$ owing to very short distance between the reference points and sources [8]. The impact of positional error on FBX measurements is expected to be quite low as it measures average volume doses unlike point dosimetry.

\section{Conclusions}

The average difference between the FBX (PT and FB) measured dose and TPS calculated average volume dose is found to be within $15 \%$. The accuracy and precision values shown with the FBX system should be reasonably sufficient for routine quality assurance in-vivo dosimetry purposes. Assuming the FB and PT as reasonable surrogates for bladder and rectum, it can be said that the FBX system can detect potential misadministrations of HDR brachytherapy in subsequent irradiation fractions. The advantage of the FBX system is its water equivalent composition, average volume dose measuring capability, energy and temperature independent response as compared to TLD or semiconductor dosimeters. However, detailed studies will be needed with regards to its safety before actual in-vivo dose measurements are possible with FBX dosimeter.

\section{References}

[1] Van Lancker M, Storme G. Prediction of severe late complications in fractionated, high dose rate brachytherapy in gynecological applications. Int J Radiat Oncol Biol Phys 1999;20:921-6.

[2] Haie-Meder C, Potter R, Limbergen EV, Briot E, De Brabandere M, Dimopoulos J, et al. Recommendations from gynaecological (GYN) GEC ESTRO working group (I): concepts and terms in 3D image-based treatment planning in cervix cancer brachytherapy- 3D dose volume parameters and aspects of 3D image-based anatomy, radiation physics, radiobiology. Radiother Oncol 2005;74:235-45.

[3] Potter R, Haie-Meder C, Limbergen EV, Barillot I, Brabandere MD, Dimopoulos J, et al. Recommendations from gynaecological (GYN) GEC ESTRO working group (II): concepts and terms in $3 \mathrm{D}$ image-based treatment planning in cervix cancer brachytherapy- 3D dose volume parameters and aspects of 3D image-based anatomy, radiation physics, radiobiology. Radiother Oncol 2006;78:67-77.

[4] Kim RY, Shen S, Duan J. Image-based three-dimensional treatment planning of intracavitary brachytherapy for cancer of the cervix: dose-volume histograms of the bladder, rectum, sigmoid colon, and small bowel. Brachytherapy 2007;6:187-94.

[5] International Commission on Radiation Units and Measurements. ICRU report 38: dose and volume specifications for reporting intracavitary therapy in gynecology. Bethesda, MD: ICRU; 1985.

[6] International Commission on Radiation Units and Measurements. ICRU report 58: dose and volume specification for reporting interstitial therapy. Bethesda, MD: ICRU; 1997.

[7] Anagnostopoulos G, Baltas D, Geretschlaeger A, Martin T, Papagiannis $P$, Tselis $N$, et al. In-vivo thermoluminescence dosimetry dose verification of transperineal ${ }^{192} \mathrm{Ir}$ high-doserate brachytherapy using CT-based planning for the treatment of prostate cancer. Int J Radiat Oncol Biol Phys 2003; 57(4):1183-91.

[8] Stuecklschweiger GF, Arin-Schad KS, Poier E, Poschauko J, Hackl A, et al. Bladder and rectal dose of gynecologic highdose-rate implants: comparison of orthogonal radiographic measurements with in-vivo and CT-assisted measurements. Radiology 1991;181:889-94.

[9] Lahtinen T, Tenhunen M, Vayrynen M. ICRU reference points and maximum doses of rectum and bladder in intracavitary radiotherapy. Radiother Oncol 1993;28:174-6.

[10] Clark B, Souhami L, Roman T, Evans M, Pla C. Rectal complications in patients with carcinoma of the cervix treated with concomitant cisplatin and external beam irradiation with high dose rate brachytherapy: a dosimetric analysis. Int J Radiat Oncol Biol Phys 1994;28:1243-50.

[11] Alecu R, Alecu M. In-vivo rectal dose measurements with diodes to avoid misadministration during intracavitary high dose rate brachytherapy for carcinoma of the cervix. Med Phys 1999;26:768-70. 
[12] Waldhausl C, Wambersie A, Potter R, George D. In-vivo dosimetry for gynaecological brachytherapy: physical and clinical considerations. Radiother Oncol 2005;77:310-7.

[13] Gupta BL. Low level dosimetry studies with the $\mathrm{FeSO}_{4}$ - benzoic acid - xylenol orange system. In: Dosimetry in agriculture, industry, biology and medicine. Vienna: International Atomic Energy Agency; 1973. p. 421-32 (IAEA-SM-160/77).

[14] Gupta BL, Kini UR, Bhat RM, Madhvanath U. Use of the FBX dosimeter for the calibration of cobalt-60 and high energy teletherapy machines. Phys Med Biol 1982;27:235-45.

[15] Gupta BL, Kini UR, Bhat RM, Madhvanath U. Intercomparison of ionization and chemical dosimetry techniques for central axis depth-dose measurements from cobalt-60 teletherapy machines. AMPI Med Phys Bull 1986;11:28-31.

[16] Gupta BL, Narayan GR, Bhat RM, Kini UR, Ramaswamy RS, Suseela $B$. Use of the FBX dosimeter for the quality assurance of ${ }^{60} \mathrm{Co}$ and high-energy teletherapy machines by mail. Phys Med Biol 1992;37:2095-102.
[17] Semwal MK, Bansal AK, Thakur PK, Vidyasagar PB. In-vivo (entrance) dose measurements in external beam radiotherapy with aqueous FBX dosimetry system. J Med Phys 2005;30(1): $32-5$.

[18] Semwal MK, Bansal AK, Thakur PK, Vidyasagar PB. FBX aqueous chemical dosimeter for measurement of virtual wedge profiles. J Appl Clin Med Phys 2008;9(4):206-10.

[19] Madhvanath U, Kini UR, Gupta BL. Calibration of brachytherapy sources using ferrous sulphate - benzoic acid - xylenol orange dosimeter. Int J Appl Radiat Isot 1976;27:443-6.

[20] Driscoll CMH. Fundamental aspects of TLD materials. In: Hufton AP, editor. Practical aspects of thermoluminescence dosimetry. Manchester: The Hospital Physicists' Association; 1984. p. 5-11.

[21] Toye W, Das R, Kron T, Franich R, Johnston P, Duchesne G. An in-vivo investigative protocol for HDR prostate brachytherapy using urethral and rectal thermoluminescence dosimetry. Radiother Oncol 2009;91:243-8. 